\title{
Influence of operating firing field technologies on operational performance parameters
}

\author{
Aleksandr Kazakov ${ }^{1}$, and Anton Suprunovskiy ${ }^{2, *}$ \\ ${ }^{1}$ Matrosov Institute for System Dynamics and Control Theory, 664033 Irkutsk, Russia \\ ${ }^{2}$ Irkutsk State Transport University, 664074 Irkutsk, Russia
}

\begin{abstract}
At present, introduction of operating firing field technologies is one of the urgent tasks of the Russian railway transport, as their use can significantly improve operational performance parameters. The factors and conditions of transition to operating firing field control system for operational performance are investigated in this paper. It provides the analysis of the problems that arise during the work of the operating firing field and proposes measures for their elimination. Methods of mathematical and computer modeling using the author's software product were used. A new approach to multilevel simulation of the work of a transport operating firing field has been developed, which involves the use of the mathematical apparatus of multiphase queuing systems.
\end{abstract}

\section{Introduction}

Russian Railways JSC is performing purposeful work for transition from the regional principles of traffic control to arrangement of train traffic in operating firing field in the network. Implementation of a new transportation process model must ensure improvement of operational performance parameters. Unified control of train flows in the operating firing field allows accelerating the traffic. It is possible to arrange an efficient logistics technology with this control type. This approach will allow managing loading and movement of cargo flows to large unloading centers and seaports [1].

The problem of estimating efficiency of the main transportation process indicators arises when moving to the operating firing field railway control system. The difficulty consists in consideration of influence of various factors in the context of their close interconnection. The problem of accounting operating costs when changing the boundaries of operating firing fields determines the need to use methods of mathematical estimates and forecasts. It is necessary to take into consideration fulfillment of the controllability conditions by such large system as the "transportation process" to solve the problem of selecting the boundaries of a control operating firing field when determining the number of operating firing fields.

The next problem is arrangement of an optimal system for building the areas of circulation of locomotive crews within the boundaries of the operating firing fields created. When allocating boundaries of operating firing fields, it shall also be necessary to take into

\footnotetext{
*Corresponding author: as.irgups@gmail.com
} 
consideration the distribution of tag control centers. The specifics of location of locomotives technical inspection points should also be taken into consideration. The criteria for creating operating firing fields were defined (see below) to solve the problems identified in the course of selection of operating firing fields boundaries. The following authors also worked on this topic: E. A. Sotnikov, A. N. Baushev, A. T. Osminin.

In determining the boundaries of operating firing fields formation, E. A. Sotnikov proposes to use the calculation methods [2]. On the basis of such methods, A. T. Osminin proposes variant of calculation of the number of operating firing fields.

Professor Osminin proposes calculation of the number of operating firing fields in the railway network by using mathematical approach. The purpose is to build the operating firing field coverage with the maximum flow connectivity and the maximum balance based on actual data on the flows of loaded cars.

As a result of the calculations, maps of the railway network with the optimal location of the operating firing fields boundaries were compiled. Variants with 5 and 6 operating firing fields were proposed [2].

The purpose of this work is to develop a conceptual approach to determine the efficiency of application of operating firing field technologies in the railway network. The following shall be necessary: to consider the criteria of transition to the operating firing field control system of the transportation process; to study the possibility of reducing the operating costs for the transportation process by improving the performance of performance indicators; to study existing problems at performance of operating firing field technologies.

The task that must be solved to achieve this goal is selection of the mathematical apparatus for simulating performance of the operating firing fields system. The mathematical model must provide an opportunity to predict performance of the system at various input parameters, including the transport flow characteristics [3]. The model will allow estimating the efficiency of improving operational performance of the transportation process. It is proposed to use a multilevel stochastic model to achieve this objective in this paper. The number of levels can be arbitrarily large, however, first, the problems discussed can be described with the help of models with a number of levels of not more than three; second, an increase in the number of levels makes the model very difficult to study [4]. A similar approach was earlier applied by the authors, in particular, for simulation of transportation and logistics systems at the regional level [5].

\section{Operating firing field creation criteria}

The analysis of performance conditions of the transportation process for the last 50 years makes enables to give a clear definition of the transportation process control operating firing field. A transportation process control operating firing field shall mean a set of sections of the network that have a single technology of traction rolling stock performance. When servicing common cargo flows, the identity of the production cycles is revealed and their improvement is achieved [6].

The study of factors and conditions for transition to the operating firing field control system for operational performance of the railway network makes it possible to establish the following basic criteria for the operating firing fields created [6]:

1. Ensuring technological completeness of the transportation process for arrangement and promoting the cars and train traffic within operating firing fields.

2. Creation of a flexible system for building locomotive circulation areas and work areas for locomotive crews within the transportation process control operating firing fields.

3. Rationalization of the development and implementation of plans for repair and construction works on the main directions of the railway network.

4. Ensuring favorable conditions for large handling areas and adjacent approaches. 
5. Ensuring convenient conditions for arrangement local performance with large flow of cars at operating firing fields.

6. Providing opportunities for railways to perform social functions related to arrangement of passenger traffic in all types of communications.

\section{Operational performance parameters in arrangement of operating firing fields}

Introduction of operating firing field technologies makes it possible to improve the quality of freight traffic and to reduce the company expenses by improving the conditions for controlling the transportation process. With that, the level of fulfillment of the main qualitative indicators of operational performance is increased and the idle time of trains and locomotives at technical stations is reduced. This is achieved by improving the quality of operational train planning by the following indicators:

- increase in sectoral speed due to increased rhythm and uniformity of train traffic;

- reduction in the number of trains that have been left behind by traffic, which must proceed to seaports and other large unloading points, through the use of logistics technologies in regulating loading and advancement of trains at operating firing fields;

- reducing the risks of violation of delivery deadlines due to a reduction in the number of trains left behind by traffic and an increase in the sectoral speed;

- improving the use of locomotives by reducing their idle time at technical stations pending the implementation of maintenance operations (TO-2) and reducing the mileage of empty runs.

Quantification of the impact of operating firing field technologies on performance indicators is of great interest. However, the values of the latter are affected by a large number of random factors [7], therefore, this task is very complicated and has not yet been solved.

\section{Justification of selection of the study method}

There is also a significant uncertainty in behavior of large system called "transportation process in the operating firing field" caused by the following factors: technical and operational failures; changing requirements and actions of clients; conditions of interaction between independent control subsystems; weather conditions; employees' errors; other impacts on the course of the transportation process.

All this makes it extremely difficult to build a rigorous common mathematical model for solving the problem under consideration, which, apparently, should be stochastic (statistical, see below) [8]. Logical analysis can be used as an alternative to mathematical simulation and expert assessments of the impact of operating firing field technology on the change in the values of performance indicators can be carried out. However, this approach is quite costly and is not universal.

Thus, despite the existing difficulties, the most promising study method is mathematical simulation using the probabilistic (stochastic) approach. We will also use other methods of systems analysis, such as analysis and synthesis, decomposition and aggregation (see the next section), in the course of the study. 


\section{Changes in operational performance parameters with the use of operating firing field technologies}

Let us consider the reasons and the nature of changes in indicators of operational performance at transition to operating firing field technologies. Idle time of trains and locomotives at technical stations. With introduction of operating firing fields technologies, the train traffic occurs more regularly due to the following:

- elimination of inter-track junctions with exception of the delivery period impact;

- preventing thickening of the working cars fleet in certain operating firing fields areas, particularly on approaches to ports;

- planning the passage of trains within the boundaries of the operating firing field, not railways.

It is known that processing of trains upon arrival and breaking-up depends on the uniformity of arrival of trains to classification yards. A uniform flow reduces the idle time of trains and cars. This applies to transit trains that are not processed. Besides, improvement of locomotive scheduling for trains makes it possible to reduce idle times while waiting for delivery of locomotives and finished trains [9].

The performance indicators are significantly improved with the use of operating firing field technologies. First of all, idle time for various operations is reduced. This is associated with the improvement in conditions of arrangement of locomotives turnover. Reducing the idle time for performing station operations also reduces the duration of holding of the station tracks, which causes their freeing. The number of freed tracks can be determined on the basis of the reduction in the time of occupying the station tracks. Freeing of arrival and departure tracks improves the maneuverability of railway operating firing fields and improves arrangement of operational performance. Reducing the idle time to departure from stations due to more regular train flows during the day provides more efficient use of the operating time of locomotives and locomotive crews. Increasing the uniformity of train traffic with the transition to operating firing field technology provides reduction in duration within daily periods, when freight trains pass in a more condensed manner. The analysis of the size of transfer of trains on inter-track junctions for the first and the second half of the day shows that freight traffic increases noticeably during the period from 6 a.m. to 6 p.m. [10].

Thus, it can be seen that performance indicators are indeed significantly increased when implementing the operating firing field technology. On the contrary, operational costs are reduced and labor productivity indicators are increasing.

\section{Mathematical model}

Selection of the mathematical apparatus for simulation of operating firing field technologies for controlling the transportation processes is determined by the following two principal circumstances. First, as already noted, considerable uncertainty exists in the system caused by various technical, operational and economic factors. Second, the three-level control of the transportation process is practiced in the operating firing field system. In this situation, the use of a three-level stochastic model, which is a multiphase queuing system from the mathematical standpoint, is evident [11].

Let us review system of random values $S, X_{1}, \ldots, X_{n}, Y_{1}, \ldots, Y_{m}$, which has the following structure:

1. "input" - random value $S$ (first level);

2. “intermediate" - random vector $\vec{X}=\left(X_{1}, \ldots, X_{n}\right)$ (second level); 
3. "output" - random vector $\vec{Y}=\left(Y_{1}, \ldots, Y_{k}\right)$ (third level).

These random variables can be either discrete or continuous.

Depending on the value of random variable $S$ at the first level, the distribution laws of random variables of the second level are defined. $X_{i}=X_{i}(S), i=1, \ldots n$. In their turn, the laws of distribution of random variables of the third level depend on the values that random variable $S 0 \mathrm{x} 1$ and random vector $\vec{X}$ take. $Y_{j}=Y_{j}\left(S, X_{1}, \ldots, X_{k}\right), j=1, \ldots k$.

With that, $S$ actually performs as the initial data and in certain cases its values may be set or uniquely determined from additional conditions; $\vec{X}$ is the vector of auxiliary parameters; $\vec{Y}$ is the vector of the main characteristics of the process studied, which are of interest from the simulation standpoint. It can easily be seen that the model considered is indeed a three-level model. The number of levels can be increased if necessary. However, the study becomes much more complicated with introduction of each new level [11].

The study of the model built is only possible using numerical methods. First of all, these are the Monte Carlo methods [12]. To summarize this section, it should be noted that the technique proposed earlier in [13] can be used to establish the boundaries of a particular operating firing field.

\section{Operational performance parameters of the railway operation in conditions of operating firing field technologies}

The Eastern Operating Firing field (EOD) is currently becoming the key region for the social and economic development of Russia in the context of activation of the eastern vector of the country's economic development. It is necessary to create real instruments that give a positive impulse to the entire transportation process for more efficient control of the operational performance of the EOD. One of such instruments is a unified model for planning performance indicators. First of all, planned values for the operating control center (OCC) of the EOD shall be established and then, they shall be established for the EOD railways. Due to this, the basic principles of the transportation process operating firing field control are preserved. This approach will allow arranging the through passage of trains with setting the uniform weight norms, reducing the number of idle periods at technical stations, reducing costs.

Let us review the results of operation for 2017. Table 1 contains the main indicators of operational performance of the entire EOD and the indicator for the railways within it.

Table 1. Operation indicators for 2017.

\begin{tabular}{|l|c|c|c|}
\hline \multicolumn{1}{|c|}{ Indicators } & Planned & Actual & $+/-$ \\
\hline Sectoral speed (km/hour) & 39.7 & 39.9 & 0.2 \\
\hline $\begin{array}{l}\text { Locomotive productivity (thousand gross tons- } \\
\mathrm{km})\end{array}$ & 2,249 & 2,261 & 12 \\
\hline Average train weight (tons) & 3,969 & 3,984 & 15 \\
\hline Daily locomotive run (km) & 723 & 728 & 5 \\
\hline Average loading (thousand tons) per day & 563.8 & 568.7 & 4.9 \\
\hline
\end{tabular}

Based on the results of 2017, the main performance indicators for implementation of operating firing field technologies are in the positive trend. The average train weight was increased by 15 tons, the average daily locomotive run was increased by $5 \mathrm{~km}$, the locomotive productivity grew by 12 thousand gross tons-km. 
The criterion for efficiency of introduction of operating firing field technologies is fulfilling the operational performance indicators: freight turnover, share of shipments delivered within the standard period, average delivery rate of freight shipments in loaded cars, average delivery rate of freight shipments in containers, route speed of a freight train, fulfillment of the freight trains schedule, implementation of the plan for unloading at port stations, implementation of the plan for delivery of trains to port stations, average daily capacity of locomotives, average train weight in tons, average daily locomotive run, quality of the arrangement of infrastructure repair works, the provision of "windows" in accordance with the directive schedule.

It is necessary to perform a preliminary scenario simulation of the EOD performance for efficient implementation of these criteria. For this purpose, it is planned to develop its simulation model based on the approach of mathematical simulation of operating firing field technologies proposed in the previous section.

\section{Results}

The following has been determined in the course of the study performed:

1. Introduction of operating firing field technologies is a promising direction for development of the Russian railway transport in general and the eastern services operating firing field in particular.

2. It is necessary to perform scenario simulation of operation of the operating firing field to determine its quantitative parameters.

3. The operating firing field is influenced by a large number of random factors, due to which its mathematical model must have the probabilistic (stochastic) nature.

4. The use of the apparatus multilevel (multiphase) queuing systems for simulation is viewed as the most promising approach in the conditions described above.

\section{Conclusion}

The prospects for development of operating firing field technologies are based on arrangement of the transportation process control within the boundaries of the railway network with the efficient use of the capacity and carrying capacity of the infrastructure, traction resources and technical means to support the transportation process. At the same time, the following functions must be performed in the operating firing field: a) control on the basis of logistic train traffic control principles; b) redistribution of car flows by directions in accordance with the shift and daily plan for train and cargo operations, the traffic schedule and the train formation plan. Based on the local conditions, a set of basic indicators of operational performance can be supplemented and modified to take into consideration the development of a system of key performance indicators of the Russian Railways JSC holding company. The mathematical model allows more detailed consideration of the relation between the operating firing field technologies control level and estimation of the effect on the state of the system when input flows change.

Further development of the studies must be related to improvement of the mathematical model and its software implementation in the form of the operating firing field simulation model with subsequent verification of the model on actual data. When implementing this idea, the tools will be obtained that will allow performing scenario simulation of application of operating firing field technologies in railway transport.

The research was performed with the financial support of the Russian Foundation for Basic Research within the scope of research project No.18-07-00604. 


\section{References}

1. E. Kreutzberger, R. Konings, Research in Transportation Business and Management, Vol. 19, 83-96 (2016).

2. A.T. Osminin, Bjulleten' Objedinennogo uchenogo soveta OAO RZD [Bulletin of the Joint Scientific Council of Russian Railways JSC], 2, 42-56 (2017). (in Russian)

3. F.A. Haight, Mathematical Theories of Traffic Flow (NY-London Academic Press, 1963).

4. G. Bolch, S. Greiner, H. de Meer, K.S. Trivedi, Queueing Networks and Markov Chains. Modeling and Performance Evaluation with Computer Science Applications (NY Wiley \& Sons, 2006).

5. I.V. Bychkov, A.L. Kazakov, A.A. Lempert, D.S. Bukharov, A.B. Stolbov, Automation and Remote Control, Vol. 77, 332-343 (2016).

6. A.T. Osminin, E.A. Sotnikov, A.N. Baushev, Zheleznodorozhnyj transport [Railway transport], 6, 21-27 (2017). (in Russian)

7. E.A. Sotnikov, Zheleznodorozhnyj transport [Railway transport], 3, 4-8 (2018). (in Russian)

8. G.J. Hahn, S.S. Shapiro, Statistical Models in Engineering (NY Wiley \& Sons, 1994).

9. D.Yu. Levin, Dispetcherskie centry i tehnologija upravlenija perevozochnym processom [Dispatching centers and control technology of the transportation process] (Marshrut, Moscow, 2005). (in Russian)

10. B.S. Kerner, Introduction to Modern Traffic Flow Theory and Control (Springer, Berlin, 2009).

11. C. Kim, A. Dudin, O. Dudina, S. Dudin, European Journal of Operational Research, Vol. 235, 170-179 (2014).

12. C. Graham, D. Talay, Stochastic Simulation and Monte Carlo Methods, Mathematical Foundations of Stochastic Simulation (Springer, 2013).

13. A.L. Kazakov, A.A. Lempert, International Journal of Artificial Intelligence, Vol. 13, 200-210 (2015). 